\title{
Aortic Dissections in the Population-Based Danish National Patient Registry from 1996-2016: A Validation Study
}

\author{
Lasse Mollegaard Obel $\mathbb{D}^{1,2}$, Jes S Lindholt $\mathbb{D}^{1,2}$, Anne N Lasota $\mathbb{D}^{3}$, Henrik K Jensen ${ }^{4,5}$, \\ Leila Louise Benhassen ${ }^{5,6}$, Amalie L Kristensen ${ }^{5,6}$, Reshaabi Srinanthalogen ${ }^{6}$, Malina Christiansen $\mathbb{D}^{7}$, \\ Henning Bundgaard ${ }^{7}$, Mads Liisberg $\mathbb{D}^{1,2}$ \\ 'Department of Cardiothoracic and Vascular Surgery, University Hospital Odense, Odense, Denmark; ${ }^{2}$ Elitary Research Centre of Individualized \\ Medicine in Arterial Disease (CIMA), University Hospital Odense, Odense, Denmark; ${ }^{3}$ Department of Vascular Surgery, Aalborg University Hospital, \\ Aalborg, Denmark; ${ }^{4}$ Department of Cardiology, Aarhus University Hospital, Aarhus, Denmark; ${ }^{5}$ Department of Clinical Medicine, Aarhus University, \\ Health, Aarhus, Denmark; ${ }^{6}$ Department of Cardiothoracic and Vascular Surgery, Aarhus University Hospital, Aarhus, Denmark; ${ }^{7}$ Department of \\ Cardiology, The Heart Center, University of Copenhagen, Copenhagen, Denmark
}

Correspondence: Lasse Mollegaard Obel, Department of Cardiothoracic and Vascular Surgery, Odense University Hospital, Denmark, Tel +45 654 I 24 02, Email Lasse.Mollegaard.Obel@rsyd.dk

Purpose: This study evaluated the validity of the ICD-10 diagnostic codes for aortic dissections (ADs) in the Danish National Patient Registry (DNPR) based upon positive predictive values (PPV).

Patients and methods: Cases registered in the DNPR with the unspecific AD diagnostic code DI710 (unspecified AD) from 1996 to 2016, and the specific AD diagnostic codes DI710A (AD Type A) and DI710B (AD Type B) from 2006 to 2016, were included. Available medical records from all registered cases underwent review. Confirmed cases of AD served as "gold standard" when reporting PPV. PPV estimates were stratified by regional differences, date, age at time of diagnosis, and sex.

Results: A total of 5018 cases were identified in the DNPR. After merging of data and retrieval of medical records, 3767 cases were eligible for validation. Of these, 2677 cases were verified as AD type A (59.7\%), AD type B (38.8\%), and unspecified type of AD (1.5\%). The average age at diagnosis was $65.1 \pm 13.0$ years $(67.3 \%$ males $)$. The overall PPV for having an AD when one of the three diagnostic codes were registered from 1996 to 2016 was 71.1\% (95\% confidence interval (CI): 69.6-72.5) and increased significantly over time. From 2006 to 2016, the PPV for the specific AD diagnostic codes was 89.5\% (95\% CI: 87.4-91.3), whilst the PPV for the unspecific diagnostic code was 63.5\% (95\% CI: 61.1-65.9).

Conclusion: We found the overall PPV for the pooled AD diagnostic codes in the DNPR acceptable. However, the two specific AD diagnostic codes presented remarkably higher PPV compared to the unspecific diagnostic code.

Keywords: aortic dissection, validation, positive predicted values, population-based, epidemiology

\section{Background}

Aortic dissections (ADs) are relatively uncommon but highly life-threatening as they may lead to systemic ischemia, organ failure, or complete rupture of the aorta. An AD develops when a tear in the intimal aortic layer causes blood to divide the arterial wall in two. This allows blood to flow in a retro- or anterograde dissection plane between the inner and medial aortic layers. ${ }^{1}$

\section{Classification of Aortic Dissections}

An AD is typically categorized according to location of entry and extent of the aorta using either DeBakey's or Stanford's classification system. ${ }^{1-3}$ This report adhered to the Stanford classification system, where an AD type A involves the ascending aorta, and an AD type B occurs distally to the left subclavian artery with no involvement of the ascending aorta. 


\section{The Danish National Patient Registry}

As ADs are relatively uncommon, the majority of research relies on data from registries. However, a shared limitation when conducting registry-based studies is the validity of data as shortcomings in coding policies may occur. This may lead to over- or under-estimation of AD cases and associated parameters. ${ }^{4,5}$

The population-based Danish National Patient Registry (DNPR) is considered to be of high quality globally. ${ }^{6}$ Data from the DNPR merged with other Danish registries, eg, the Cause of Death registry, enables unique, large epidemiological studies on common as well as on rare diseases. The ICD-10 classification system was implemented in the DNPR as of $1^{\text {st }}$ of January $1994 .^{6}$ However, the initial ICD-10 codes did not distinguish between the different types of AD. Therefore, the only available ICD-10 code from 1994-2005 for AD was "unspecified AD" (DI710). The specific diagnostic codes for AD type A and type B, ie, DI710A and DI710B, were added to the revised ICD-10 codebook in $2006 .^{7}$

Before conducting studies based upon data derived from the DNPR, quality and usability of data need to be assessed and validated. This has yet to be done for the AD diagnostic codes.

Consequently, the aims for this study were: i) to evaluate the overall validity of data from the DNPR by reporting positive predictive values (PPV) for the primary AD diagnostic codes registered at time of admission at a Danish hospital in the period from 1996 to 2016, ii) to report PPV for the specific AD diagnostic codes registered from 2006 to 2016, and iii) to report PPV for the unspecific diagnostic code registered from 2006 to 2016, using medical records as gold standard references.

\section{Methods}

\section{Setting}

Denmark has a total of 5.8 million inhabitants (January 2021), and the health care system is organized in five regions: The Capital Region of Denmark (1.9 million), the Region of Zealand (0.8 million), the Region of Southern Denmark (1.2 million), the Region of Central Denmark (1.3 million), and the Region of Northern Denmark (0.6 million). ${ }^{8}$ The Danish National Health Service facilitates tax-supported health care for all Danish residents including acute emergency services, procedures, and surgeries. This study encompasses and validates primary AD diagnostic codes registered in the DNPR from the year 1996 to 2016 from all five Danish regions. The study was approved by the Danish Data protection agency and the Danish patient safety authority (Identification number: 3-3013-2537/1) leading to exemption for ethical approval as declared in the Danish health law (paragraph 46, section 2).

\section{The Central Personal Registration System}

In Denmark, a Central Personal Registration System was established in 1968 assigning a unique 10-digit civil registration number (CPR-number) to each Danish citizen. The registry contains individual information on sex, date of birth, place of birth and residence, citizenship, marital status, and the identity of parents and spouses. ${ }^{9}$ The CPR-number enables matching of data from different databases and registries at an individual level.

\section{The Danish National Patient Registry}

The DNPR was established in 1977 and provides information from all hospital contacts including hospital-based outpatient care. Hence, primary and secondary diagnoses provided from hospitalizations, date and time of admission, hospital and departments, medical procedures, interventions, and operations are registered in the DNPR. This information is linked with the patient's unique CPR-number. ${ }^{10}$ The registration of diagnostic codes into the DNPR is permorfed by medical staff including physicians and secretaries. Every Danish hospital is obligated to submit records to the DNPR monthly, ensuring up-to-date data.

\section{Study Population}

This validation study evaluated data from all Danish patients registered in the DNPR with a primary AD diagnostic code in the period of 1996 to 2016. More specifically, all patients who received an AD diagnosis at time of admission to a 
Danish hospital in the given time span with either ICD-10 DI710 (unspecified AD), DI710A (AD Type A), or DI710B (AD Type B) were included and validated. It is noteworthy that the unspecific AD diagnostic code has been used throughout the whole study-period, whereas the specific AD diagnostic codes were not available until the year 2006 and forward.

\section{Validation}

Medical records were retrieved and reviewed by one of seven competent vascular surgeons, vascular surgeon trainees, or cardiothoracic surgeon trainees. Medical records were evaluated thoroughly from a pre-defined protocol. First, surgery descriptions by cardiologists, vascular-, or cardiothoracic-surgeons were identified and reviewed. Secondly, computed tomography scans or magnetic resonance imagining descriptions by vascular radiologists were reviewed. After review, each case was classified as an "AD type A", "AD type B", or "verified, but unspecified type of AD". If no surgery or imaging description suggested $\mathrm{AD}$, the case was classified as "no $\mathrm{AD}$ ". In uncertain cases, a secondary review was performed by one of the other six reviewers. Data were entered and stored in an encrypted SharePoint, and later merged with data from the DNPR and the CPR-registry at a remote desktop hosted by Statistics Denmark.

\section{Statistical Analyses}

The diagnostic codes registered in the DNPR were divided into three categories: AD registered by any of the three diagnostic codes for AD in the period 1996-2016, the specific AD diagnostic codes (ICD-10 DI710A and ICD-10 DI710B) registered from 2006-2016, and the non-specific AD diagnostic code (ICD-10 DI710) registered from 2006-2016.

If a specific AD diagnostic code was misregistered in the DNPR as AD type A instead of type B or vice versa, based upon the medical record review, the case was excluded from the study.

The PPV was chosen as measure of diagnostic validity and was computed by dividing confirmed AD cases from medical records with AD cases registered in the DNPR. This was done for all three categories of AD diagnostic codes mentioned previously. All PPVs are reported with 95\% confidence intervals $(95 \% \mathrm{CI})$.

For evaluation of potential differences within subgroups, secondary analyses were performed. Despite the Danish healthcare system being considered homogenous across regions, ${ }^{11}$ this study provides PPV for AD from each of the five regions separately. Furthermore, calculation of PPVs was stratified by date, age at time of diagnosis, and sex. Potential differences in PPV within the subgroups were tested with Pearson's Chi-squared test, and p-values $<0.05$ were considered significant. All analyses were performed with STATA/IC 16.1 (StataCorp, College Station, TX, USA).

\section{Results}

A total of 5018 cases were registered in the DNPR from 1996-2016 with a primary AD diagnostic code at time of admission. Matching of data at an individual level between the DNPR and the CPR-registry was unsuccessful in 397 cases $(7.9 \%)$, leading to a study population of 4621 registered cases. Of these, medical records were un-retrievable in 806 cases (16.1\%). Upon review of the remaining 3815 medical records, 48 cases (1.0\%) were excluded due to misclassification in the DNPR as AD type A instead of AD type B or vice versa. Consequently, the final study population eligible for validation included 3767 cases of $A D$ registered in the DNPR (Figure 1).

Distribution of the diagnostic codes in the DNPR in the final study population for unspecific AD, type A, and type B was $72.5 \%, 15.9 \%$, and $11.6 \%$, respectively.

A total of 2677 cases $(67.3 \%$ males) were verified as $\mathrm{AD}$ from medical records' review. Distribution of the verified cases of AD type A and type B was $59.7 \%$ and $38.8 \%$ ( $<<0.001$ ), respectively, and unspecified type of AD was $1.5 \%$ (Figure 1). Based upon the validated cases, the overall mean age at time of dissection was $65.1 \pm 13.0$ years, and the mean age for AD type A and type B was $63.4 \pm 13.1$ years and $67.5 \pm 12.4$ years $(\mathrm{p}<0.001)$, respectively. 


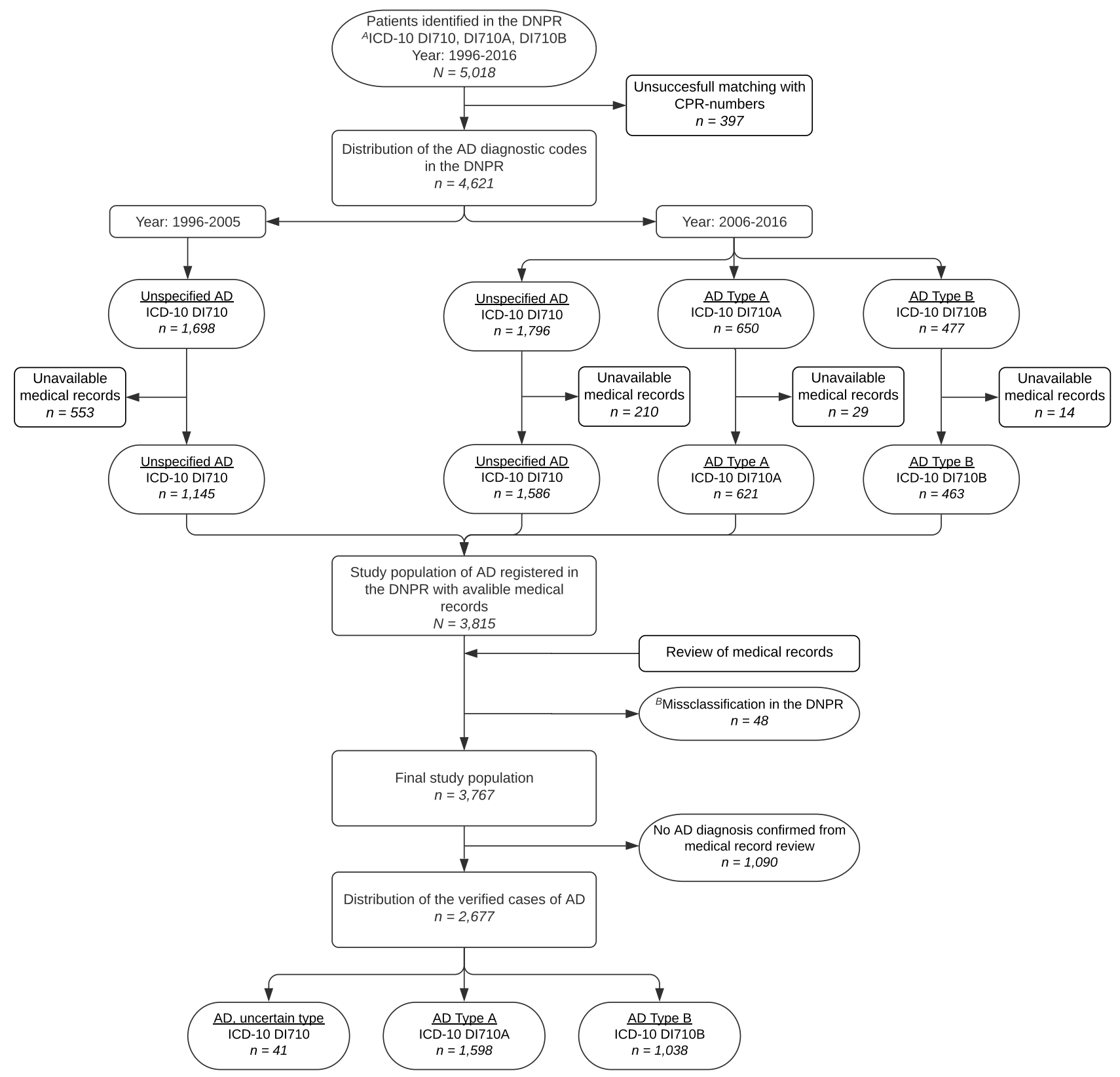

Figure I Flowchart of the validation process including distributions of diagnostic codes in both the DNPR and the validated cases.

Notes: ${ }^{A}$ Unspecified aortic dissection (DI7I0), aortic dissection type A (DI7IOA), aortic dissection type B (DI7IOB); ${ }^{B}$ cases registered in the DNPR with a specific diagnostic code, ie, DI7IOA or DI7IOB, but validated as the opposite type of AD than registered, were excluded from the final study population. The 48 excluded cases were 25 and 23 cases of $A D$ type $A$ and type $B$, respectively.

Abbreviations: AD, aortic dissection; DNPR, Danish National Patient Registry.

\section{Primary Analyses}

According to Table 1, the overall PPV for having an AD when any one of the three diagnostic codes were registered in the DNPR from 1996-2016 was 71.1\% (95\% CI: 69.6-72.5). The PPV increased significantly over time ranging from 64.9\% (95\% CI: 60.6-69.1) in the period 1996-2000 to 78.0\% (95\% CI: 75.9-79.9) in the period 2011-2016 (p=0.009).

As can be seen in Table 2, the overall PPV for having an AD when a specific AD diagnostic code was registered from 2006-2016, ie, ICD-10 DI710A and ICD-10 DI710B, was 89.5\% (95\% CI: 87.4-91.3). The PPV for AD type A and type B was 90.3\% (95\% CI: 87.6-92.6) and 88.1\% (84.7-91.0) $(\mathrm{p}=0.79)$ respectively, and no significant changes in PPV were observed from 2006-2010 to 2011-2016 for either of the two specific diagnostic codes. 
Table I Validity of Aortic Dissection Diagnostic Codes in the DNPR from 1996-2016

\begin{tabular}{|c|c|c|c|c|}
\hline \multirow[b]{3}{*}{ Overall } & \multicolumn{4}{|c|}{$\begin{array}{l}\text { Registered Cases of AD in the DNPR } \\
\text { Year: } 1996-2016\end{array}$} \\
\hline & Confirmed AD & DNPR & PPV (95\% Cl) & $\mathbf{P}$ \\
\hline & 2677 & 3767 & $71.1 \%(69.6-72.5)$ & - \\
\hline \multicolumn{5}{|l|}{ Date } \\
\hline$-1996-2000$ & 324 & 499 & $64.9 \%(60.6-69.1)$ & \multirow[t]{4}{*}{0.009} \\
\hline$-200 I-2005$ & 419 & 646 & $64.9 \%(61.0-68.5)$ & \\
\hline$-2006-2010$ & 646 & 970 & $66.6 \%(63.5-69.6)$ & \\
\hline$-2011-2016$ & 1288 & 1652 & $78.0 \%(75.9-79.9)$ & \\
\hline
\end{tabular}

Notes: Positive predicted values (PPVs) of diagnostic codes for aortic dissections (ADs) registered in the Danish National Patient Registry (DNPR) from I996 to 2016, using medical records as "gold standard" references. In this table, no stratifications between the three different diagnostic codes were made. The P-value was derived from Pearson's Chi-squared test and test for difference in PPV between the different time periods. A p-value $<0.05$ is considered significant.

Abbreviations: $\mathrm{AD}$, aortic dissection; $\mathrm{Cl}$, confidence interval; DNPR, the Danish National Patient Registry; PPV, positive predicted value.

The overall PPV for having an AD when the unspecific diagnostic code was registered in the DNPR from 2006-2016, ie, ICD-10 DI710, was 63.5\% (95\% CI: 61.1-65.9) and increased significantly from $60.6 \%$ (95\% CI: $57.1-64.1)$ to $66.1 \%$ (95\% CI: 62.8-69.4) from 2006-2010 to 2011-2016 ( $\mathrm{p}=0.023)$, respectively.

\section{Secondary Analyses}

The PPV for the specific AD diagnostic codes displayed little differences across the geographically different regions from 2006 to 2016, despite a lower PPV for AD type A in the Region of Zealand. Some differences in PPV between the regions were observed when the unspecific diagnostic code was registered; the lowest PPV was $42.3 \%$ and the highest $68.6 \%$ (Supplementary Table 1).

In all age groups, the overall PPVs for the specific diagnostic codes were between $86.5 \%$ and $93.5 \%$ (Supplementary Table 2). For AD type B, the PPV was significantly different within age groups, with the lowest PPV of $83.8 \%$ observed in the group of patients over 70 years old. The overall PPV for the unspecific AD diagnostic code in all age groups varied from $58.4 \%$ to $71.9 \%$ and was equally significantly lower in the group of patients over 70 years old compared to the younger age groups.

When the specific AD diagnostic codes were registered, the overall PPVs were high within both males and females with no significant differences between sexes, whilst the unspecific diagnostic codes presented a lower PPV for AD in both males and females (Supplementary Table 2).

\section{Discussion}

\section{Main Findings}

In this comprehensive, nationwide study, validity of the three ICD-10 diagnostic codes for AD was evaluated by reporting PPVs after review of medical records. Overall, based upon review of medical records from 3767 registered cases in the DNPR from 1996-2016, we confirmed 2677 cases of AD. The overall PPV for AD when a patient was registered with one of the three diagnostic codes from 1996 to 2016 was 71.1\%, whilst the overall PPV from 2006-2016 for the specific diagnostic codes was $89.5 \%$ and the unspecific diagnostic code was $63.5 \%$, respectively.

$\mathrm{AD}$ type $\mathrm{A}$ was twice as common as type $\mathrm{B}$, and type $\mathrm{A}$ was twice as common in males than females, supporting findings from previous studies. ${ }^{1,12,13}$ The mean age of 65.1 years at time of dissection supports that an $\mathrm{AD}$ occurs more frequently in patients over 60 years old. ${ }^{12,13}$ 
Table 2 Validity of the Specific and Unspecific Aortic Dissection Diagnostic Codes in the DNPR from 2006-2016

\begin{tabular}{|c|c|c|c|c|c|c|c|c|}
\hline \multirow[b]{3}{*}{ Overall } & \multicolumn{4}{|c|}{$\begin{array}{l}\text { Specific AD Diagnostic Codes in the DNPR } \\
\text { Year: 2006-2016 } \\
\text { AD Type A (DI7 IOA)/AD Type B (DI7 IOB) }\end{array}$} & \multicolumn{4}{|c|}{$\begin{array}{c}\text { Unspecific AD Diagnostic Code in the DNPR } \\
\text { Year: 2006-2016 } \\
\text { Unspecified AD (DI7 I0) }\end{array}$} \\
\hline & Confirmed AD & DNPR & PPV (95\% Cl) & $\mathbf{P}$ & Confirmed AD & DNPR & PPV (95\% CI) & $\mathbf{P}$ \\
\hline & 927 & 1036 & $89.5 \%(87.4-91.3)$ & & 1007 & 1586 & $63.5 \%(6 I .1-65.9)$ & \\
\hline Type A & 540 & 598 & $90.3 \%(87.6-92.6)$ & \multirow[t]{2}{*}{0.79} & 611 & - & - & \\
\hline Type B & 386 & 438 & $88.1 \%(84.7-91.0)$ & & 373 & - & - & \\
\hline Unspecified $A D^{A}$ & I & - & - & & 23 & - & - & \\
\hline \multicolumn{9}{|l|}{ Date } \\
\hline \multicolumn{9}{|l|}{$A D$ overall ${ }^{B}$} \\
\hline$-2006-2010$ & 184 & 208 & $88.5 \%(83.3-92.5)$ & \multirow[t]{2}{*}{0.59} & 462 & 762 & $60.6 \%(57.1-64.1)$ & \multirow[t]{2}{*}{0.023} \\
\hline$-2011-2016$ & 743 & 828 & $89.7 \%$ (87.5-91.7) & & 545 & 824 & $66.1 \%(62.8-69.4)$ & \\
\hline \multicolumn{9}{|l|}{ AD Type A } \\
\hline$-2006-2010$ & 95 & 109 & $87.2 \%(79.4-92.8)$ & \multirow[t]{2}{*}{0.22} & 270 & - & - & - \\
\hline$-2011-2016$ & 445 & 489 & $91.0 \%(88.1-93.4)$ & & 341 & - & - & - \\
\hline \multicolumn{9}{|l|}{ AD Type $B$} \\
\hline$-2006-2010$ & 88 & 99 & $88.9 \%(81.0-94.3)$ & \multirow[t]{2}{*}{0.79} & 176 & - & - & - \\
\hline$-2011-2016$ & 298 & 339 & $87.9 \%(84.0-9 \mid .2)$ & & 197 & - & - & - \\
\hline
\end{tabular}

Notes: Positive predicted values (PPVs) of diagnostic codes for aortic dissections (ADs) registered in the Danish National Patient Registry (DNPR) from 2006 to 2016, using medical records as "gold standard" references. PPVs were stratified by the specific and unspecific diagnostic codes. P-values were derived from Pearson's Chi-squared test and test for difference in PPV between the different time periods. A p-value $<0.05$ is considered significant and written in bold. ${ }^{A}$ An unspecified aortic dissection was registered when an aortic dissection was validated in the medical records, but it was not possible to determine specific type of dissection. ${ }^{B}$ The overall $A D$ includes the unspecified validated dissections.

Abbreviations: AD, aortic dissection; Cl, confidence interval; DNPR, The Danish National Patient Registry; PPV, positive predicted value.

\section{The Overall PPV from 1996-2016}

Evaluating all three diagnostic codes, the average PPV in the DNPR from 1996-2016 was considered acceptable. The PPV increased significantly over time, which is most likely caused by two factors; first, the specific AD diagnostic codes were not available before 2006. When the specific diagnostic codes for AD were implemented, the physician was obligated to commit to the $\mathrm{AD}$ diagnosis and to distinguish between the two types of $\mathrm{AD}$. In order to discriminate between AD type A and type B, a full truncal computed tomography scan or magnetic resonance imaging scan is most often required, leading to the second potential factor improving the PPV over time. Development in emergency rooms when handling patients with possible aortic catastrophes with fast diagnostic imaging accuracy has increased over time, leading to an initially more precise registration of diagnostic code into the DNPR.

\section{The Specific Aortic Dissection Diagnostic Codes from 2006-2016}

This study confirmed a high number of $\mathrm{AD}$ cases from medical records when the specific AD diagnostic codes were registered from 2006 to 2016, ie, ICD-10 DI710A and DI710B, yielding high validity of data. This implies that further studies based upon the specific primary AD diagnostic codes from the DNPR will be reliable in terms of diagnostic accuracy. The PPV varied little between AD type A and type B, respectively, besides a significantly lower PPV for AD type B in patients more than 70 years old compared to the younger age groups. Furthermore, the nationwide high PPV 
confirms that the Danish healthcare system is homogenous across regions. ${ }^{11}$ Additionally, the consistently high PPV in date indicates that the DNPR is a valid source for identification of patients with previous events of AD.

\section{The Unspecific Aortic Dissection Diagnostic Code from 2006-2016}

The validity of data was lower when the unspecific AD diagnostic code, ie, ICD-10 DI710, was registered from 2006 to 2016 compared to the specific AD diagnostic codes. This is not surprising and is most likely caused by the speculative origin of the "unspecific" term. When a patient arrives at an emergency department, they are registered with a tentative diagnosis. The main symptom of an $\mathrm{AD}$ is abrupt onset of chest and/or back pain. ${ }^{1}$ However, this symptom may be present in various other conditions including aortic aneurysm rupture, acute myocardial infarction, facet joint syndrome, and more. Furthermore, the relatively lower PPV for the unspecified AD diagnostic code may be due to lack of verification of the $\mathrm{AD}$ from imaging, insufficient descriptions from radiologists, or that the staff coding the $\mathrm{AD}$ were not involved adequately in the given patient-case upon registration. However, a significant increase in PPV over time for the unspecific AD diagnostic code was observed, which may be due to the positive development in fast diagnostic imaging.

\section{Limitations and Strengths}

This was the first study to evaluate validity of the AD diagnostic codes in the DNPR. A prominent strength of this validation study is the inclusion and validation of data from all five Danish regions of Denmark, ensuring data quality across the country. Furthermore, validations of medical records were performed according to a standardized protocol by competent reviewers, ensuring accurate validation and minimizing risk of inter-observer variability.

An inherent limitation when evaluating a large amount of data cross-sectionally from a registry is the loss of data due to unsuccessful merging with CPR-numbers, unlocatable medical records, or lack of fully described events in medical records. In this study, $24 \%$ of the registered cases in the DNPR were not included due to missing data. An additional $1 \%$ was excluded from the study population due to misclassification in the DNPR when registered as a specific aortic dissection but with wrong location. However, we were able to access and adequately evaluate a high number of medical records, making results reasonably reliable. Furthermore, it should be noted that medical competencies vary between the staff making the registrations to the DNPR, as both physicians and secretaries register the ICD-10 codes. This may influence the overall quality of the database.

Finally, this study did not include undiagnosed cases of AD. This led to missing calculations of sensitivity, specificity, and negative predicted values.

\section{Conclusion}

This study found an overall PPV of 71.1\% for the pooled AD diagnostic codes registered in the DNPR from 1996 to 2016, and the PPV increased significantly over time. However, it is noteworthy that from 2006-2016, the PPVs for the specific $\mathrm{AD}$ diagnostic codes were remarkably higher compared to the unspecific $\mathrm{AD}$ diagnostic code. Hence, the specific AD diagnostic codes ICD-10 DI710A and DI710B are reliable in terms of diagnostic accuracy, whereas the unspecific AD diagnostic code ICD-10 DI710 should be trusted more cautiously.

\section{Author Contributions}

All authors made a significant contribution to the work reported, whether that is in the conception, study design, execution, acquisition of data, analysis and interpretation, or in all these areas; took part in drafting, revising or critically reviewing the article; gave final approval of the version to be published; have agreed on the journal to which the article has been submitted; and agree to be accountable for all aspects of the work.

\section{Funding}

This study was supported by the University of Southern Denmark, the Helse foundation, and the Region of Southern Denmark. 


\section{Disclosure}

HKJ is supported by grants from the Novo Nordisk Foundation, Denmark (NNF18OC0031258 and NNF20OC0065151) and received lecture fees from Abbott, Denmark and Biosense Webster, Europe. The authors report no other conflicts of interest in this work.

\section{References}

1. Erbel R, Aboyans V, Boileau C, et al. 2014 ESC Guidelines on the diagnosis and treatment of aortic diseases: document covering acute and chronic aortic diseases of the thoracic and abdominal aorta of the adult. The Task Force for the Diagnosis and Treatment of Aortic Diseases of the European Society of Cardiology (ESC). Eur Heart J. 2014;35(41):2873-2926. doi:10.1093/eurheartj/ehu281

2. DeBakey ME, McCollum CH, Crawford ES, et al. Dissection and dissecting aneurysms of the aorta: twenty-year follow-up of five hundred twentyseven patients treated surgically. Surgery. 1982;92(6):1118-1134.

3. Hagan PG, Nienaber CA, Isselbacher EM, et al. The International Registry of Acute Aortic Dissection (IRAD): new insights into an old disease. JAMA. 2000;283(7):897-903. doi:10.1001/jama.283.7.897

4. Reutersberg B, Salvermoser M, Trenner M, et al. Hospital incidence and in-hospital mortality of surgically and interventionally treated aortic dissections: secondary data analysis of the nationwide german diagnosis-related group statistics from 2006 to 2014. J Am Heart Assoc. 2019;8(8): e011402. doi:10.1161/JAHA.118.011402

5. Smedberg C, Steuer J, Leander K, Hultgren R. Sex differences and temporal trends in aortic dissection: a population-based study of incidence, treatment strategies, and outcome in Swedish patients during 15 years. Eur Heart J. 2020;41(26):2430-2438. doi:10.1093/eurheartj/ehaa446

6. Lynge E, Sandegaard JL, Rebolj M. The danish national patient register. Scand J Public Health. 2011;39(7 Suppl):30-33. doi:10.1177/ 1403494811401482

7. The Danish Health Data Authority. SKS-classification codebook, 2006. Available from: https://sundhedsdatastyrelsen.dk/da/rammer-og-retningslin jer/om-klassifikationer/sks-klassifikationer/download-sks. Accessed December 6, 2021.

8. Statistics Denmark. Available from: https://www.statistikbanken.dk/. Accessed February 5, 2021.

9. Schmidt M, Pedersen L, Sørensen HT. The danish civil registration system as a tool in epidemiology. Eur J Epidemiol. 2014;29(8):541-549. doi:10.1007/s10654-014-9930-3

10. Schmidt M, Schmidt SA, Sandegaard JL, Ehrenstein V, Pedersen L, Sorensen HT. The Danish National Patient Registry: a review of content, data quality, and research potential. Clin Epidemiol. 2015;7:449-490. doi:10.2147/CLEP.S91125

11. Henriksen DP, Rasmussen L, Hansen MR, Hallas J, Pottegard A. Comparison of the Five Danish Regions Regarding Demographic Characteristics, Healthcare Utilization, and Medication Use-A Descriptive Cross-Sectional Study. PLoS One. 2015;10(10):e0140197. doi:10.1371/journal. pone. 0140197

12. Chan KK, Rabkin SW. Increasing prevalence of hypertension among patients with thoracic aorta dissection: trends over eight decades-a structured meta-analysis. Am J Hypertens. 2014;27(7):907-917. doi:10.1093/ajh/hpt293

13. Lemaire SA, Russell L. Epidemiology of thoracic aortic dissection. Nat Rev Cardiol. 2011;8(2):103-113. doi:10.1038/nrcardio.2010.187

Clinical Epidemiology

\section{Publish your work in this journal}

Clinical Epidemiology is an international, peer-reviewed, open access, online journal focusing on disease and drug epidemiology, identification of risk factors and screening procedures to develop optimal preventative initiatives and programs. Specific topics include: diagnosis, prognosis, treatment, screening, prevention, risk factor modification, systematic reviews, risk \& safety of medical interventions, epidemiology \& biostatistical methods, and evaluation of guidelines, translational medicine, health policies \& economic evaluations. The manuscript management system is completely online and includes a very quick and fair peer-review system, which is all easy to use. 